\title{
Unbiased Monte Carlo cluster updates with autoregressive neural networks
}

\author{
Dian Wu ${ }^{\circ},{ }^{*}$ Riccardo Rossi, ${ }^{\dagger}$ and Giuseppe Carleo $\mathbb{0}^{\dagger}$ \\ Institute of Physics, École Polytechnique Fédérale de Lausanne (EPFL), CH-1015 Lausanne, Switzerland
}

(Received 28 May 2021; accepted 20 October 2021; published 10 November 2021)

\begin{abstract}
Efficient sampling of complex high-dimensional probability distributions is a central task in computational science. Machine learning methods like autoregressive neural networks, used with Markov chain Monte Carlo sampling, provide good approximations to such distributions but suffer from either intrinsic bias or high variance. In this Letter, we propose a way to make this approximation unbiased and with low variance. Our method uses physical symmetries and variable-size cluster updates which utilize the structure of autoregressive factorization. We test our method for first- and second-order phase transitions of classical spin systems, showing its viability for critical systems and in the presence of metastable states.
\end{abstract}

DOI: 10.1103/PhysRevResearch.3.L042024

\section{INTRODUCTION}

Markov chain Monte Carlo (MCMC) [1] is an unbiased numerical method that allows sampling from unnormalized probability distributions, a central task in many areas of computational science. MCMC is commonly used, for example, in molecular dynamics [2], as well as statistical and quantum physics [3-6]. In addition to fundamental applications, MCMC serves as a physics-inspired approach to solve a variety of computational problems, including combinatorial optimization [7,8] and computer graphics [9]. While MCMC is a generically applicable technique, its implementation can be plagued by long mixing or autocorrelation time [10]. Various techniques have been proposed to increase the efficiency of MCMC [11], for example, cluster updates [12,13], parallel tempering [14], the worm algorithm [15], and event-chain Monte Carlo [16]. However, these faster MCMC algorithms rely on details of the physical system considered, and they cannot be applied generically.

Machine learning (ML) methods, given their intrinsic flexibility in addressing problems in computational physics [17], are being intensively investigated as a way to improve MCMC. Applications in this direction include, for example, self-learning Monte Carlo methods [18-23], enhanced sampling driven by neural networks [24,25], and neural importance sampling (NIS) [26]. Strongly rooted in the principles of statistical physics, variational sampling techniques are among the most promising ML-driven approaches. Generative neural samplers (GNSs) [27-29] are a chief example of MLdriven variational methods. These approaches build on the

\footnotetext{
*dian.wu@epfl.ch

†riccardo.rossi@epfl.ch

${ }^{\ddagger}$ giuseppe.carleo@epfl.ch
}

Published by the American Physical Society under the terms of the Creative Commons Attribution 4.0 International license. Further distribution of this work must maintain attribution to the author(s) and the published article's title, journal citation, and DOI. idea of constructing approximate representations of the original probability distribution at hand. The resulting variational approximations can efficiently perform sampling by construction, thus completely bypassing MCMC. A particularly interesting aspect of this approach is its systematic improvability when using the free energy bound minimization as the guiding principle to gauge the approximation accuracy. The main drawback of the variational approach, however, is that the estimators of expectation values are intrinsically biased by the representation error of the approximated distribution. As unbiased estimators are of central importance in many fundamental applications in physics, recent research has started addressing the key problem of removing the bias induced by ML variational representations, for example, through importance sampling, and incorporating again MCMC strategies $[26,30,31]$.

In this Letter, we propose a way to combine variational techniques with MCMC by using autoregressive neural networks $[32,33]$ to propose cluster updates. We first show that existing unbiased sampling schemes using global updates proposed by GNS can be plagued by the ergodicity issue due to the generic presence of "exponentially suppressed configurations (ESCs)," which have a limited effect on the variational free energy but a rather strong effect on the autocorrelation time. Our workaround to this problem consists of two ingredients. On one hand, we consider physical symmetry operations that leave the Hamiltonian invariant. When applied to the MCMC states, these symmetry operations significantly reduce the exponential suppression of configurations belonging to the same equivalence class. On the other hand, we take advantage of the structure of autoregressive factorization to propose MCMC updates with clusters of spins. The cluster update scheme is automatically learned for any Hamiltonian and is therefore particularly helpful for Hamiltonians with no known cluster update scheme. We benchmark our technique on the two-dimensional Ising model, showing that our solution eliminates the ergodicity issue of the global update approach in the critical region. We then study an Ising-like frustrated plaquette model (FPM) for which traditional cluster algorithms 
are not applicable, and we find a first-order transition from a paramagnetic (PM) state to a "ferrimagnetic" (fM) state that breaks the $\mathbb{Z}_{2} \times \mathbb{Z}_{2} \times \mathbb{Z}_{2}$ symmetry of the Hamiltonian. We show that the method greatly alleviates the metastability issue, as it can rapidly thermalize by cluster updates.

\section{A. Bias in neural sampling}

In the following, we consider a system of $V$ classical Ising spins $\mathbf{s}:=\left(s_{1}, \ldots, s_{V}\right), s_{i} \in\{-1,1\}$, at inverse temperature $\beta$. We use a GNS $q_{\theta}$ with parameters $\theta$ that variationally approximates the Boltzmann probability distribution $p(\mathbf{s}) \propto$ $\tilde{p}(\mathbf{s}):=e^{-\beta E(\mathbf{s})}$ by minimizing, in the language of statistical physics, a free energy bound [27], which is equivalent to minimizing the Kullback-Leibler (KL) divergence [34]:

$$
D_{\mathrm{KL}}\left(q_{\theta} \| p\right):=\sum_{\mathbf{s}} q_{\theta}(\mathbf{s}) \ln \frac{q_{\theta}(\mathbf{s})}{p(\mathbf{s})} .
$$

To construct an expressive $q_{\theta}$, we use an autoregressive neural network to decompose it into a product of conditional probabilities $q_{\theta}(\mathbf{s})=: \prod_{i=1}^{V} q_{\theta ; i}\left(s_{i} \mid \mathbf{s}_{<i}\right)$, where $\mathbf{s}_{<i}:=$ $\left(s_{1}, \ldots, s_{i-1}\right)$. This specific choice for the model allows us to efficiently sample from the distribution $q_{\theta}(\mathbf{s})$ by sampling from the conditional probabilities $\left\{q_{\theta ; i}\right\}$ sequentially [27].

The variational autoregressive approach is systematically improvable and allows exact sampling. However, the fact that the two distributions are only approximately equal $q_{\theta}(s) \approx$ $\tilde{p}(\mathbf{s})$ also implies that the samples $\left\{\mathbf{s}^{(1)}, \ldots, \mathbf{s}^{(N)}\right\}$ drawn from the network carry an intrinsic bias. When these samples are used to compute the expectation value of an observable, the resulting estimator $\bar{O}=\frac{1}{N} \sum_{i=1}^{N} O\left(\mathbf{s}^{(i)}\right)$ is biased, and most importantly, it is not possible in general to reliably estimate the direction and the magnitude of such bias.

\section{B. NIS and global updates}

References. [30,31] have proposed two closely related solutions to the bias problem. The first method, which we denote NIS in the following, consists of using the modified unbiased estimator $\bar{O}=\sum_{i=1}^{N} w\left(\mathbf{s}^{(i)}\right) O\left(\mathbf{s}^{(i)}\right)$, where $w\left(\mathbf{s}^{(i)}\right):=$ $\frac{\tilde{w}\left(\mathbf{s}^{(i)}\right)}{\sum_{j=1}^{N} \tilde{w}\left(\mathbf{s}^{(j)}\right)}$ and $\tilde{w}\left(\mathbf{s}^{(i)}\right):=\frac{\tilde{p}\left(\mathbf{s}^{(i)}\right)}{q_{\theta}\left(\mathbf{s}^{(i)}\right)}$ are the normalized and the unnormalized weights, respectively. The second proposed solution, which we denote neural global updates (NGUs) hereafter, consists of using the GNS as a MCMC proposer: if $\mathbf{s}$ is the Markov chain state, a proposed state $\mathbf{s}^{\prime}$ is drawn from the GNS and accepted with the Metropolis probability:

$$
P_{\mathrm{acc}}\left(\mathbf{s} \rightarrow \mathbf{s}^{\prime}\right):=\min \left[1, \frac{\tilde{w}\left(\mathbf{s}^{\prime}\right)}{\tilde{w}(\mathbf{s})}\right]
$$

\section{METHODS}

\section{A. ESCs}

We point out an elementary property of the KL divergence, Eq. (1): the cost of allowing a single bad approximation $q_{\theta}(\mathbf{s})$ scales only logarithmically with the ratio $q_{\theta}(\mathbf{s}) / p(\mathbf{s})$. Therefore, it is reasonable to expect that, even when the free energy is well approximated after the variational training, $q_{\theta}(\mathbf{s})$ is still exponentially smaller than $p(\mathbf{s})$ for a small portion of configurations. We call them ESCs. ${ }^{1}$ We denote $p_{\text {ESC }}$ to be the probability that a configuration is an ESC. ${ }^{2}$ A well-trained network has $p_{\mathrm{ESC}} \ll 1$, and they have a limited effect on the variational free energy but a rather strong effect on the autocorrelation time.

Let us consider a Markov chain evolution using NGUs and suppose that the Markov chain state $\mathbf{s}$ is an ESC. The ratio $\tilde{w}\left(\mathbf{s}^{\prime}\right) / \tilde{w}(\mathbf{s})$ in Eq. (2) will be exponentially small for almost any other configuration $\mathbf{s}^{\prime}$; therefore, the Markov chain will be essentially stuck in $\mathbf{s}$ for a long time before accepting any new proposal, and the autocorrelation time of the whole chain will be impractically large. A similar argument applies when considering the variance of the NIS method.

\section{B. Symmetry-enforcing updates}

To solve the generic ergodicity problem of NGU methods, we start by proposing an enhanced MCMC method to enforce the symmetries. At each Monte Carlo step, we apply a random element of the symmetry group $G$, composed of a translation and reflections, to the current configuration. ${ }^{3}$ There is no need to reject this action because the energy is invariant under the action. In the following, we refer to this method as NGUs with symmetries (NGUSs).

Assume that the current configuration $\mathbf{s}$ is an ESC, and we use a random symmetry operation to change $\mathbf{s}$ to another configuration $\mathbf{s}^{*}$ in the equivalence class $\mathcal{C}$. The probability that all configurations in $\mathcal{C}$ are ESCs is on the order of $p_{\mathrm{ESC}}^{\# \mathcal{C}}$, so it is extremely unlikely to get stuck within the equivalence class. The occurrence of ESCs does not depend on the physical symmetries but rather the structure of the network.

\section{Neural cluster updates}

With autoregressive neural networks, it is particularly natural to consider cluster updates where only a subset of the lattice is changed. Indeed, for any given $k$, it is possible to propose an update $\mathbf{s} \rightarrow \mathbf{s}^{\prime}$ by setting $\mathbf{s}_{\leqslant V-k}^{\prime}:=\mathbf{s}_{\leqslant V-k}$ and only sample $\mathbf{s}_{>V-k}^{\prime}$. The weight ratio in Eq. (2) becomes $\frac{\tilde{w}\left(\mathbf{s}^{\prime}\right)}{\tilde{w}(\mathbf{s})}=$ $\frac{\tilde{p}\left(\mathbf{s}^{\prime}\right)}{\tilde{p}(\mathbf{s})} \prod_{i=V-k+1}^{V} \frac{q_{\theta ; i}\left(s_{i} \mid \mathbf{s}_{<i}\right)}{q_{\theta i i}\left(s_{i}^{\prime} \mid \mathbf{s}_{<i}^{\prime}\right)}$, which is not too far from 1 when $k$ is small. In this way, the new configuration is closer to the old one and is easier to be accepted, so we expect lower autocorrelation time than global update methods. In the following, we refer to this method as neural cluster updates (NCUs).

As symmetry-enforcing and cluster updates are compatible with each other, we use the two at the same time, and we call the resulting method NCUs with symmetries (NCUSs), which still falls into the category of MCMC. As described in Alg. I, we randomly choose a cluster size $k$ and consider the

\footnotetext{
${ }^{1}$ In the context of variational inference, it was empirically found [35] that $q$ tends to cover fewer modes than $p$ in the probability landscape. However, the problem of ESCs is more general, as it is present even when all the modes are represented.

${ }^{2} \mathrm{~A}$ distribution of $\tilde{w}$ is shown in Fig. S3 in the Supplemental Material [36], which can be used to estimate $p_{\text {ESC }}$.

${ }^{3}$ Formally, this is equivalent to multiplying the Markov transition matrix by another matrix that leaves the equilibrium distribution invariant, as discussed in the Supplemental Material [36].
} 
Algorithm I. A step of NCUS.

\begin{tabular}{ll}
\hline \hline 1: & Input the current configuration $\mathbf{s}$ \\
2: & Sample an integer $k \in\{1, \ldots, V\}$ from $P_{\text {cluster }}$ \\
3: & Sample the last $k$ spins and propose the configuration $\mathbf{s}^{\prime}$ \\
4: & Accept $\mathbf{s} \leftarrow \mathbf{s}^{\prime}$ with probability $P_{\text {acc }}\left(\mathbf{s} \rightarrow \mathbf{s}^{\prime}\right)$ \\
5: & Translate $\mathbf{s}$ by a random displacement \\
6: & Reflect $\mathbf{s}$ along the $x$ axis, $y$ axis, and the diagonal, \\
7: & Reflect $\mathbf{s}$ along the $z$ axis (flip all spins) with $50 \%$ probability \\
8: & Output $\mathbf{s}$ as a sample in the Markov chain \\
\hline \hline
\end{tabular}

last $k$ spins $\mathbf{S}_{>V-k}$ in the autoregressive order ${ }^{4}$ to be inside the cluster. Then we sample those spins from the approximate distribution $q_{\theta}$ of the physical system learned into the network, which is conditioned on the spin configuration $\mathbf{s}_{\leqslant V-k}$ outside the cluster. After that, we accept the new configuration $\mathbf{s}^{\prime}$ with the probability $P_{\text {acc }}\left(\mathbf{s} \rightarrow \mathbf{s}^{\prime}\right)$, then randomly apply the symmetry operations. See Fig. 1 for a schematic illustration.

Although the cluster size $k$ can come from an arbitrary distribution $P_{\text {cluster }}(k)$, numerical experiments have shown that the uniform distribution $P_{\text {cluster }}(k) \equiv 1 / V$ already works better than many other cases we have explored. ${ }^{5}$

\footnotetext{
${ }^{4}$ The autoregressive order is a one-dimensional labeling $s_{k}$ of the spins in the lattice, mapped from the two-dimensional labeling $s_{i, j}$, where $k=(i-1) \times L+j$.

${ }^{5}$ A comparison of different choices of $P_{\text {cluster }}$ can be found in Figs. S1 and S2 in the Supplemental Material [36].
}

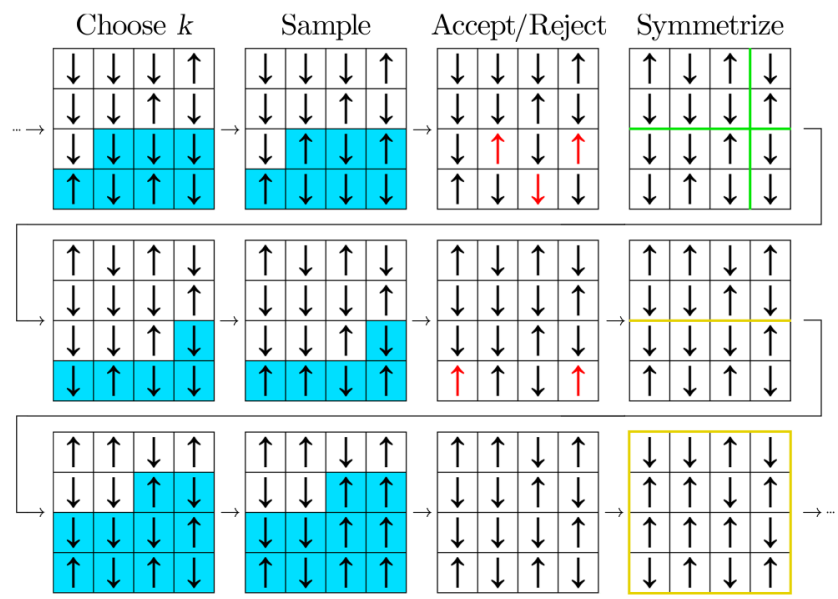

FIG. 1. Example of three steps of neural cluster updates with symmetries (NCUSs) applied to a $4 \times 4$ spin model. The columns correspond to different lines in Alg. I. The last $k$ spins that can be flipped are highlighted in blue. If a proposal is accepted, the spins actually flipped are shown in red. For translations, the original borders of the lattice are shown in green. For reflections, the plane of reflection is shown in yellow, and yellow borders around the lattice indicate a reflection along the $z$ axis (across the $x y$ plane).

\section{NUMERICAL EXPERIMENTS}

\section{A. Ising model}

We start to demonstrate the effectiveness of NCUSs on the conventional two-dimensional Ising model:

$$
E(\mathbf{s}):=\sum_{i, j=1}^{L} s_{i, j}\left(s_{i+1, j}+s_{i, j+1}\right),
$$

with periodic boundary conditions $s_{L+1, j}=s_{1, j}, s_{i, L+1}=s_{i, 1}$. The model can be solved exactly and has a critical point at $\beta=\ln (1+\sqrt{2}) / 2 \approx 0.44$ [37].

Our network architecture is based on PixelCNN [38], combined with dilated convolutions [39] to reduce the total number of parameters. Overall, our networks are lightweight and have three convolutional layers and $\sim 4 \times 10^{3}$ parameters. Thanks to the MCMC bias removal, we do not need the network to approximate the true distribution to extremely high precision, which in any case will be increasingly difficult for larger lattices. As we use the same network for all the experiments, we can compare the performances of the various unbiased sampling methods. After training the network, we generate $10^{3}$ Markov chains in parallel, each containing $10^{5}$ samples. ${ }^{6}$

When comparing the efficiencies of different MCMC algorithms, the main metric is the integrated autocorrelation time $\tau,{ }^{7}$ which determines the variance of the estimator when the variance of the observable and the sample size are given. Here, $\tau$ is an intrinsic property of the algorithm and the physical system, without dependence on the sample size, if we have enough samples to obtain a converged estimation of it. For NIS, the autocorrelation time is equal to one by definition; however, there is an increased variance arising from the reweighting procedure, which we consider an effective autocorrelation time for the sake of comparison with the other techniques.

From Fig. 2(a), we see that both NGUs and NIS have pathologically high autocorrelation times in the critical region. An inspection of their autocorrelation function [see Fig. 2(b)] shows that the Markov chain of NGUs is essentially nonergodic in the available simulation time. By contrast, our proposed method NCUSs has no issue in the critical region. A closer inspection of the inset of Fig. 2(a) shows that the autocorrelation time of NCUSs still increases in the critical region, and the sampling efficiency is improved typically by two orders of magnitude compared with the global update methods. The performance of NCUSs is also comparable with the celebrated Wolff cluster update method [13], which is specifically tailored for the Ising model. Both NCUSs and NGUSs perform well in the critical region, and NCUSs are to be preferred, as the cluster update allows us to achieve a lower autocorrelation time and, more importantly, a better asymptotic behavior of the autocorrelation function.

\footnotetext{
${ }^{6}$ Details of the network structure, training, and sampling are described in the Supplemental Material [36].

${ }^{7}$ For completeness, we provide the definitions of the autocorrelation function and the integrated autocorrelation time in the Supplemental Material [36].
} 

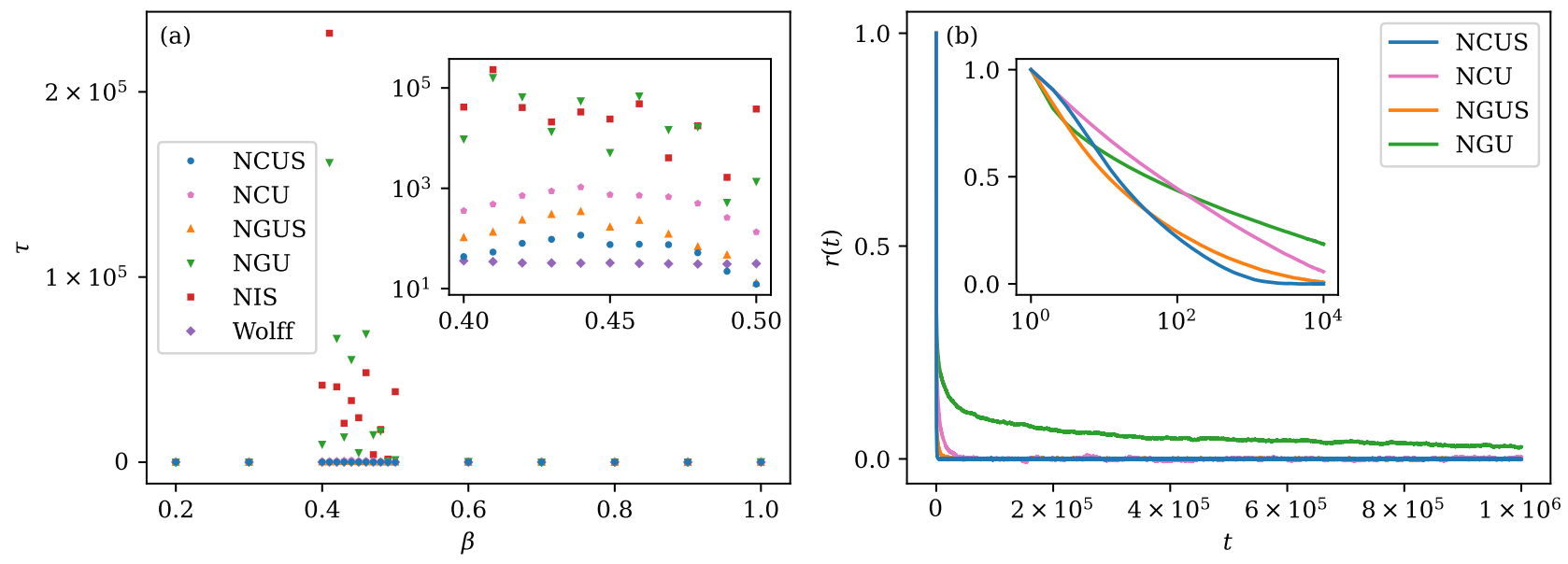

FIG. 2. (a) Integrated autocorrelation time $\tau$ as a function of temperature on the $16 \times 16$ Ising model. For neural importance sampling (NIS), we use the increased variance from the reweighting procedure as the effective autocorrelation time. The inset focuses on their behaviors near the critical point and uses the logarithmic scale on the $y$ axis. (b) Autocorrelation functions $r(t)$ on the $16 \times 16$ Ising model at $\beta=0.44$. The inset uses the logarithmic scale on the $x$ axis to focus on their behaviors at small $t$.

\section{B. FPM}

We now study another model that presents a richer physics than the Ising model and for which, to our knowledge, no traditional cluster update method is applicable. We consider a classical spin- $\frac{1}{2}$ system with nearest-neighbor $J_{1}$, next-nextnearest-neighbor $J_{3}$, and plaquette $K$ interactions:

$$
\begin{aligned}
E(\mathbf{s}):= & J_{1} \sum_{i, j=1}^{L} s_{i, j}\left(s_{i+1, j}+s_{i, j+1}\right) \\
& +J_{3} \sum_{i, j=1}^{L} s_{i, j}\left(s_{i+2, j}+s_{i, j+2}\right) \\
& +K \sum_{i, j=1}^{L} s_{i, j} s_{i+1, j} s_{i, j+1} s_{i+1, j+1},
\end{aligned}
$$

with periodic boundary conditions, which we denote as the FPM. In this Letter, we set $J_{1}=J_{3}=-1$ and $K>0$.

We sketch the expected phase diagram in Fig. 3(a). The ground state of the FPM depends on the competition of $J_{1}$ and $K$. For small $K$, we expect a transition as a function of temperature between PM and ferromagnetic (FM) phases, which is analogous to what is found in the conventional Ising model. For $K>1$, the ground state is a repetition of a $2 \times 2$ unit cell containing one spin pointing in the opposite direction of the other three spins, as shown in Fig. 3(a). The ground state breaks the $\mathbb{Z}_{2}$ spin-inversion symmetry and the $\mathbb{Z}_{2} \times \mathbb{Z}_{2}$ translation symmetry of one lattice spacing in the $x$ and $y$ directions. As this phase has an average magnetization per site of $\frac{1}{2}$, we refer to this phase as fM. At finite temperature, there must be a phase transition between the PM phase and the fM one, the nature of which we investigate in this Letter.

We present the numerical results for the energy per site $\varepsilon$ and the spontaneous magnetization per site $m:=\left\langle\left|\frac{1}{V} \sum_{i} s_{i}\right|\right\rangle$ in Figs. 3(b) and 3(c), respectively, as functions of temperature with $K=2$ and lattice sizes up to $L=32$. Our results in Fig. 3(b) strongly suggest that, in the thermodynamic limit $L \rightarrow \infty$, the energy is discontinuous at the critical point $\beta_{c}=0.2145 \pm 0.0012$ with a latent heat $Q=1.36 \pm 0.20$, estimated using the standard finite-size scaling procedure of Ref. [40]. Another indication of the first-order nature of the phase transition comes from the spontaneous magnetization shown in Fig. 3(c), which when extrapolated to the thermodynamic limit shows a discontinuity of the spontaneous magnetization from 0 to a value close to $\frac{1}{2}$, as expected for the fM phase. ${ }^{8}$

The comparison of autocorrelation times from different sampling methods is presented in Fig. 4, which provides numerical evidence that NCUSs greatly alleviate the metastability issue expected near first-order phase transitions [44-47]. Theoretically, a first-order phase transition occurs when the distribution of energy $p(E) \propto N(E) e^{-\beta E}$ has two peaks with the same size, as shown in Fig. 5, where $N(E)$ is the number of configurations with energy $E$. A GNS-based sampling method has equal probabilities to generate a sample from the two peaks, and the probability to accept that proposal will be close to one, if the network is ideally trained and there is no problem of ESC. Meanwhile, for traditional local-update MCMC methods, they can only move small horizontal steps in Fig. 5, so it takes more steps $\left(\sim L^{2}\right)$ and exponentially lower probability $\left(\sim e^{-\beta \delta E L^{2}}\right)$ for them to walk from the low-energy peak to the high-energy one, where $\delta E$ is the typical energy difference in a local update, which does not scale with $L$. In other words, the exponentially large number of configurations in the high-energy peak will not make it easier for localupdate MCMC methods to sample from that peak because it is exponentially hard for the walker to walk between those configurations in locally connected paths. NCUSs reach a balance between the two extremes, which solves the problem of ESC and keeps the autocorrelation time practically low, even

\footnotetext{
${ }^{8} \mathrm{~A}$ naive Ginzburg-Landau approach for a three-component $\mathbb{Z}_{2} \times$ $\mathbb{Z}_{2} \times \mathbb{Z}_{2}$-symmetric order parameter predicts a second-order transition when truncated at the quartic level. A first-order phase transition is also found in the $q=8$ Potts model [41-43], but the broken symmetry group there is $\mathbb{Z}_{8}$.
} 

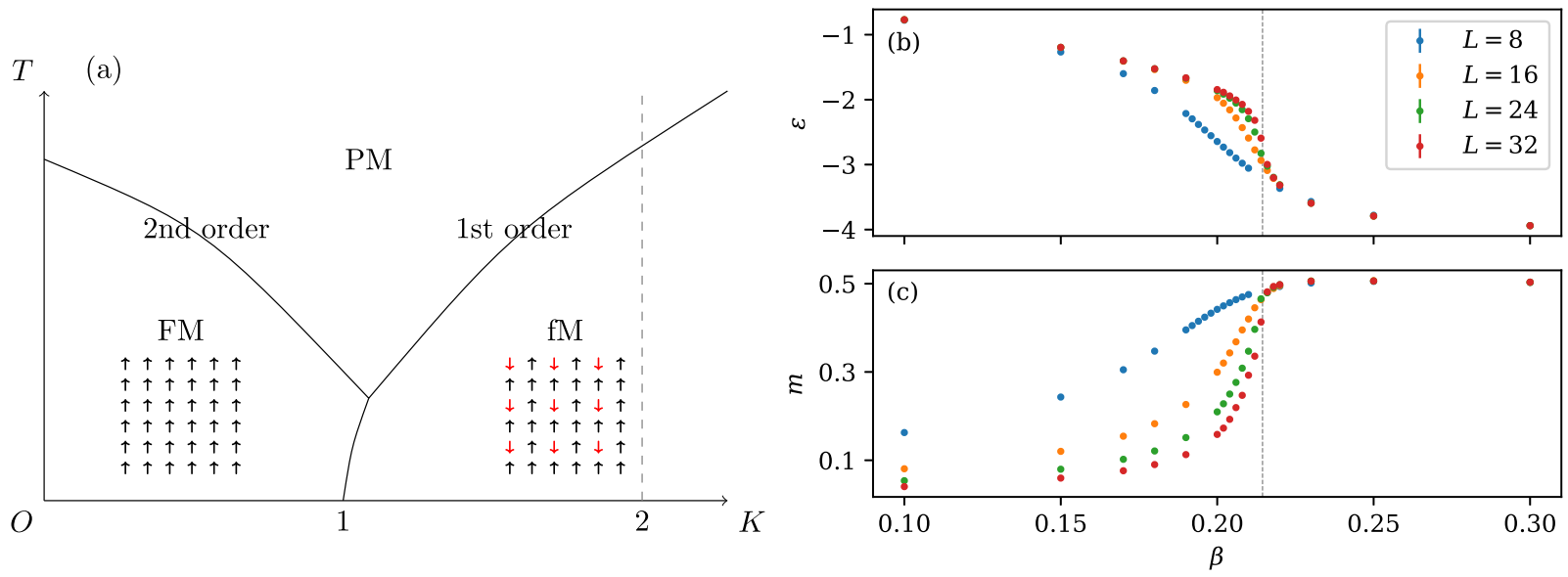

FIG. 3. (a) Sketch of the expected phase diagram of the frustrated plaquette model (FPM) for $J_{1}=J_{3}=-1$ with example ground states in the ferromagnetic (FM) and the ferrimagnetic (fM) phases. The dashed vertical line represents the temperature cut we numerically study. (b) Energy per site $\varepsilon$ and (c) spontaneous magnetization per site $m$ of the FPM for $J_{1}=J_{3}=-1, K=2$ as functions of temperature with lattice sizes up to $L=32$, obtained by neural cluster updates with symmetries (NCUSs). The dashed vertical line indicates the estimated transition point $\beta_{c}$.

if the network is lightweight and cannot ideally approximate the true distribution.

Another potential issue in first-order phase transitions is the strong divergence of the specific heat, resulting in high variance of the energy. Despite this, NCUSs still help us estimate the energy with high accuracy, and the error bars in Figs. 3(b) and 3(c) are too small to be visible.

\section{CONCLUSIONS}

In this Letter, we have shown a strategy to systematically remove the bias of variational autoregressive neural network methods and, at the same time, keep the variance of observables under control. Our approach exploits the autoregressive structure of the models to generate cluster Monte Carlo updates. After having shown that global updates proposed from networks trained with the KL divergence are generically expected to fail because of a small number of ESCs, we have provided a workaround that takes advantage of enforcing the symmetries of the physical system and from using the chainlike graphical structure of the autoregressive model, namely, NCUSs, to help the Markov chain rapidly escape from ESCs. We have benchmarked our technique for the two-dimensional Ising model, showing its efficacy in the critical region, where a straightforward implementation of NGUs fails. We have further shown the potential of our method for systems for which no traditional cluster updates are known by considering a frustrated plaquette Ising model, where we were able to determine the first-order nature of a PM-fM phase transition breaking a $\mathbb{Z}_{2} \times \mathbb{Z}_{2} \times \mathbb{Z}_{2}$ symmetry, remarking that the automatic cluster updates we used allowed us to alleviate the metastability issue.
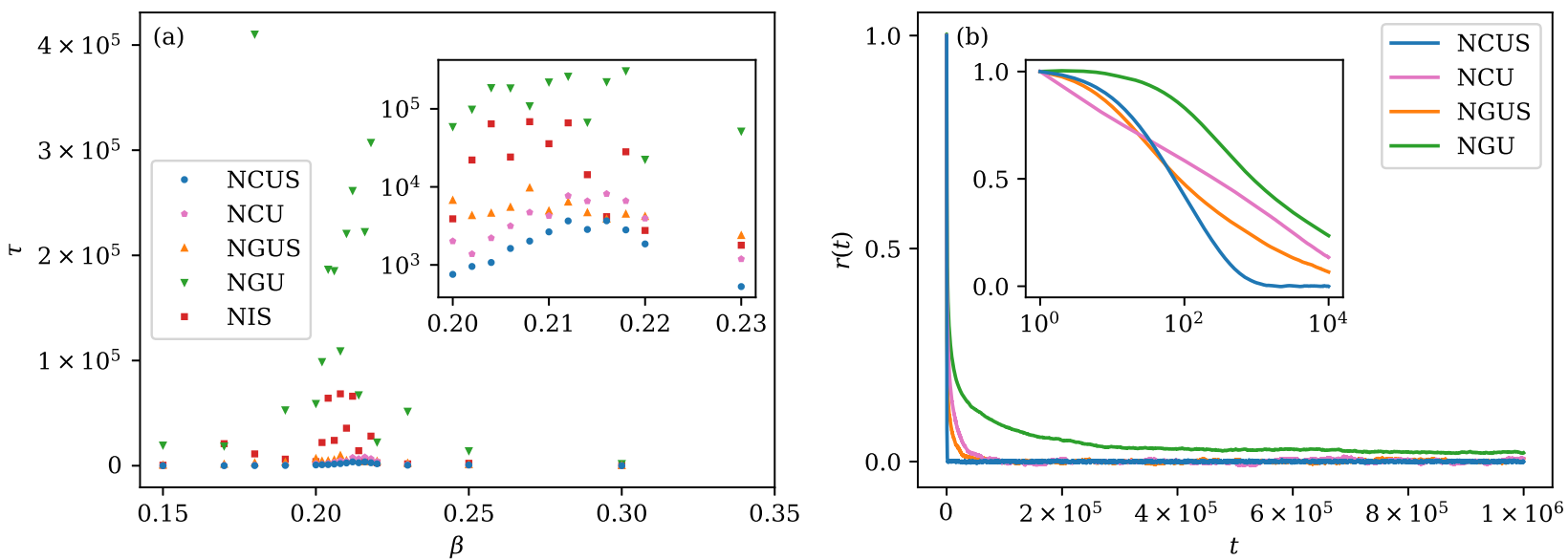

FIG. 4. (a) Integrated autocorrelation time $\tau$ as a function of temperature on the $32 \times 32$ frustrated plaquette model (FPM). For neural importance sampling (NIS), we use the increased variance from the reweighting procedure as the effective autocorrelation time. The inset focuses on their behaviors near the critical point and uses the logarithmic scale on the $y$ axis. (b) Autocorrelation functions $r(t)$ on the $32 \times 32$ FPM at $\beta=0.2$. The inset uses the logarithmic scale on the $x$ axis to focus on their behaviors at small $t$. 


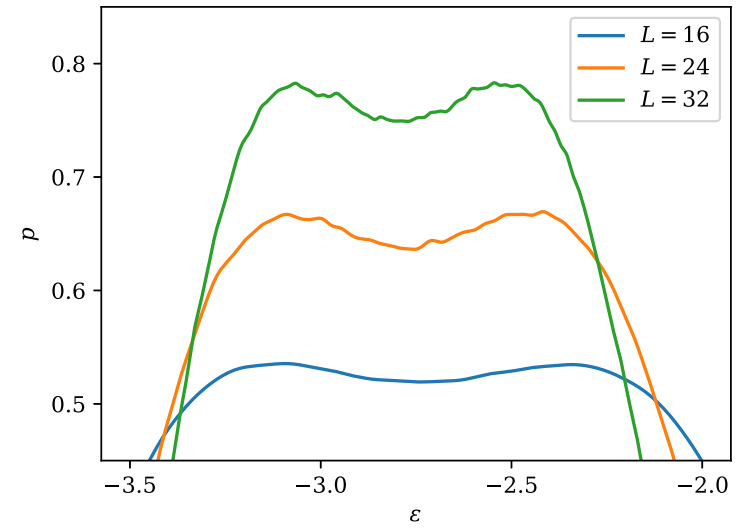

FIG. 5. Probability distribution of the energy per site $p(\varepsilon)$ for the frustrated plaquette model (FPM) with different lattice sizes $L$ at their respective phase transition temperatures, obtained by neural cluster updates with symmetries (NCUSs).

While we have been mainly concerned with the metric of autocorrelation time, we recognize that the wall-clock time is another important metric for practical computations.
In this respect, when computing the energy of the system has a negligible computational cost, current neural networkbased methods are not yet competitive with traditional MCMC methods. It can then be argued that the ideal application scenario for ML-based methods are those cases where evaluating the integrand is expensive, e.g., in determinant quantum Monte Carlo [48] and lattice field theory [49]. In future work, computational efficiency can be addressed on multiple fronts, for example, by introducing techniques such as hierarchy and sparsity of the neural network models, to reduce the computation time and scale up the lattice size by orders of magnitude. After that, we expect that the slow asymptotic growth of the autocorrelation time of GNSs will eventually make them outperform traditional MCMC methods in terms of wall-clock time.

\section{ACKNOWLEDGMENTS}

We acknowledge insightful comments and suggestions from Lei Wang and Pan Zhang. Support from the Swiss National Science Foundation is acknowledged under Grant No. 200021_200336. The computing power is supported with Cloud TPUs from Google's TensorFlow Research Cloud. Our code is available at Ref. [50].
[1] N. Metropolis, A. W. Rosenbluth, M. N. Rosenbluth, A. H. Teller, and E. Teller, Equation of state calculations by fast computing machines, J. Chem. Phys. 21, 1087 (1953).

[2] K. Binder, Monte Carlo and Molecular Dynamics Simulations in Polymer Science (Oxford University Press, Oxford, 1995).

[3] K. Binder and D. W. Heermann, Monte Carlo Simulation in Statistical Physics (Springer, Berlin, Heidelberg, 2010).

[4] W. Krauth, Statistical Mechanics: Algorithms and Computations (Oxford University Press, Oxford, 2006).

[5] J. Gubernatis, N. Kawashima, and P. Werner, Quantum Monte Carlo Methods (Cambridge University Press, Cambridge, 2016).

[6] F. Becca and S. Sorella, Quantum Monte Carlo Approaches for Correlated Systems (Cambridge University Press, Cambridge, 2017).

[7] S. Kirkpatrick, C. D. Gelatt, and M. P. Vecchi, Optimization by simulated annealing, Science 220, 671 (1983).

[8] R. Y. Rubinstein and D. P. Kroese, The Cross-Entropy Method: A Unified Approach to Combinatorial Optimization, MonteCarlo Simulation and Machine Learning (Springer Science \& Business Media, New York, 2004).

[9] R. L. Cook, Stochastic sampling in computer graphics, ACM Trans. Graph. 5, 51 (1986).

[10] H. Müller-Krumbhaar and K. Binder, Dynamic properties of the Monte Carlo method in statistical mechanics, J. Stat. Phys. 8, 1 (1973).

[11] J. S. Liu, Monte Carlo Strategies in Scientific Computing (Springer Science \& Business Media, New York, 2004).

[12] J.-S. Wang and R. H. Swendsen, Cluster Monte Carlo algorithms, Physica A 167, 565 (1990).
[13] U. Wolff, Collective Monte Carlo Updating for Spin Systems, Phys. Rev. Lett. 62, 361 (1989).

[14] R. H. Swendsen and J.-S. Wang, Replica Monte Carlo Simulation of Spin-Glasses, Phys. Rev. Lett. 57, 2607 (1986).

[15] N. V. Prokof'ev, B. V. Svistunov, and I. S. Tupitsyn, "worm" algorithm in quantum Monte Carlo simulations, Phys. Lett. A 238, 253 (1998).

[16] E. P. Bernard, W. Krauth, and D. B. Wilson, Event-chain Monte Carlo algorithms for hard-sphere systems, Phys. Rev. E 80, 056704 (2009).

[17] G. Carleo, I. Cirac, K. Cranmer, L. Daudet, M. Schuld, N. Tishby, L. Vogt-Maranto, and L. Zdeborová, Machine learning and the physical sciences, Rev. Mod. Phys. 91, 045002 (2019).

[18] D. Levy, M. D. Hoffman, and J. Sohl-Dickstein, Generalizing Hamiltonian Monte Carlo with neural networks, International Conference on Learning Representations, Vancouver, BC, Canada (2018).

[19] J. Song, S. Zhao, and S. Ermon, A-NICE-MC: Adversarial training for MCMC, Advances in Neural Information Processing Systems, Long Beach, CA (2017), pp. 5140-5150.

[20] M. Medvidovic, J. Carrasquilla, L. E. Hayward, and B. Kulchytskyy, Generative models for sampling of lattice field theories, arXiv:2012.01442 (2020).

[21] J. Liu, Y. Qi, Z. Y. Meng, and L. Fu, Self-learning Monte Carlo method, Phys. Rev. B 95, 041101(R) (2017).

[22] L. Huang and L. Wang, Accelerated Monte Carlo simulations with restricted Boltzmann machines, Phys. Rev. B 95, 035105 (2017).

[23] H. Shen, J. Liu, and L. Fu, Self-learning Monte Carlo with deep neural networks, Phys. Rev. B 97, 205140 (2018). 
[24] L. Bonati, Y.-Y. Zhang, and M. Parrinello, Neural networksbased variationally enhanced sampling, Proc. Natl. Acad. Sci. USA 116, 17641 (2019).

[25] F. Noé, S. Olsson, J. Köhler, and H. Wu, Boltzmann generators: sampling equilibrium states of many-body systems with deep learning, Science 365, eaaw1147 (2019).

[26] T. Müller, B. McWilliams, F. Rousselle, M. Gross, and J. Novák, Neural importance sampling, ACM Trans. Graph. 38, 1 (2019).

[27] D. Wu, L. Wang, and P. Zhang, Solving Statistical Mechanics Using Variational Autoregressive Networks, Phys. Rev. Lett. 122, 080602 (2019).

[28] M. S. Albergo, G. Kanwar, and P. E. Shanahan, Flow-based generative models for Markov chain Monte Carlo in lattice field theory, Phys. Rev. D 100, 034515 (2019).

[29] S.-H. Li and L. Wang, Neural Network Renormalization Group, Phys. Rev. Lett. 121, 260601 (2018).

[30] K. A. Nicoli, S. Nakajima, N. Strodthoff, W. Samek, K.-R. Müller, and P. Kessel, Asymptotically unbiased estimation of physical observables with neural samplers, Phys. Rev. E 101, 023304 (2020).

[31] B. McNaughton, M. V. Milošević, A. Perali, and S. Pilati, Boosting Monte Carlo simulations of spin glasses using autoregressive neural networks, Phys. Rev. E 101, 053312 (2020).

[32] B. Uria, M.-A. Côté, K. Gregor, I. Murray, and H. Larochelle, Neural autoregressive distribution estimation, J. Mach. Learn. Res. 17, 7184 (2016).

[33] H. Larochelle and I. Murray, The neural autoregressive distribution estimator, in International Conference on Artificial Intelligence and Statistics (2011), Vol. 15, pp. 29-37.

[34] S. Kullback and R. A. Leibler, On information and sufficiency, Ann. Math. Stat. 22, 79 (1951).

[35] I. Goodfellow, Y. Bengio, and A. Courville, Deep Learning (MIT Press, Cambridge, 2016), Chap. 3.1.3.

[36] See Supplemental Material at http://link.aps.org/supplemental/ 10.1103/PhysRevResearch.3.L042024 for a discussion about the decomposition of transition matrix, a comparison of cluster size distributions $P_{\text {cluster }}$, the definitions of autocorrelation times, details of numerical experiments, a plot of the occurrence of ESCs, and the system size dependence of autocorrelation time.
[37] L. Onsager, Crystal statistics. I. A two-dimensional model with an order-disorder transition, Phys. Rev. 65, 117 (1944).

[38] A. van den Oord, N. Kalchbrenner, and K. Kavukcuoglu, Pixel recurrent neural networks, in International Conference on Machine Learning (PMLR, New York, 2016).

[39] F. Yu and V. Koltun, Multi-scale context aggregation by dilated convolutions, in International Conference on Learning Representations (San Juan, Puerto Rico, 2016).

[40] K. Vollmayr, J. D. Reger, M. Scheucher, and K. Binder, Finite size effects at thermally-driven first order phase transitions: A phenomenological theory of the order parameter distribution, $\mathrm{Z}$. Phys. B: Condens. Matter 91, 113 (1993).

[41] H. Arisue and K. Tabata, First order phase transition of the $q$-state Potts model in two dimensions, in Non-Perturbative Methods and Lattice QCD (World Scientific, Singapore, 2001), pp. 233-241.

[42] V. Gorbenko, S. Rychkov, and B. Zan, Walking, weak firstorder transitions, and complex CFTs, J. High Energy Phys. 10 (2018) 108.

[43] V. Gorbenko, S. Rychkov, and B. Zan, Walking, weak firstorder transitions, and complex CFTs II. Two-dimensional Potts model at $Q>$ 4, SciPost Phys. 5, 050 (2018).

[44] R. Gheissari and E. Lubetzky, Mixing times of critical 2D Potts models, arXiv:1607.02182 (2016).

[45] F. Krzakala and L. Zdeborová, Hiding Quiet Solutions in Random Constraint Satisfaction Problems, Phys. Rev. Lett. 102, 238701 (2009).

[46] F. Krzakala, M. Mézard, F. Sausset, Y. F. Sun, and L. Zdeborová, Statistical-Physics-Based Reconstruction in Compressed Sensing, Phys. Rev. X 2, 021005 (2012).

[47] J. Banks, R. Kleinberg, and C. Moore, The Lovász theta function for random regular graphs and community detection in the hard regime, SIAM J. Comput. 48, 1098 (2019).

[48] R. Blankenbecler, D. J. Scalapino, and R. L. Sugar, Monte Carlo calculations of coupled boson-fermion systems. I, Phys. Rev. D 24, 2278 (1981).

[49] D. C. Hackett, C.-C. Hsieh, M. S. Albergo, D. Boyda, J.-W. Chen, K.-F. Chen, K. Cranmer, G. Kanwar, and P. E. Shanahan, Flow-based sampling for multimodal distributions in lattice field theory, arXiv:2107.00734 (2021).

[50] https://github.com/wdphy16/neural-cluster-update. 